\title{
3 Research Square

\section{Pertussis Toxin Induced Inflammatory Response Exacerbates Intracerebral Hemorrhage and Ischemic Stroke in Mice}

\section{Ming Zou}

Tianjin Medical University General Hospital

\section{Yan Li}

Tianjin Medical University General Hospital

Hui Zhao

Tianjin Medical University General Hospital

Yan Feng

Tianjin Medical University General Hospital

\section{Ying Zhang}

Tianjin Medical University General Hospital

\section{Haowen Li}

Beijing Tiantan Hospital

Qingfang Chen

Beijing Tiantan Hospital

Jingli Cao

Beijing Tiantan Hospital

\section{Shaohong Wen}

Beijing Tiantan Hospital

\section{Wenyan He}

Beijing Tiantan Hospital

Wei-Na Jin ( $\nabla$ weina.jin@ncrcnd.org.cn )

Tianjin Medical University General Hospital https://orcid.org/0000-0002-2196-9078

\section{Research}

Keywords: Brain injury, intracerebral hemorrhage, ischemic stroke, pertussis toxin, systemic inflammation

Posted Date: December 18th, 2020

DOI: https://doi.org/10.21203/rs.3.rs-126762/v1 
License: (c) (i) This work is licensed under a Creative Commons Attribution 4.0 International License. Read Full License 


\section{Abstract}

Background: Stroke is a devastating and debilitating disease and is a leading cause of death worldwide, including intracerebral hemorrhage (ICH) and ischemic stroke. Emerging evidence indicates that inflammatory cascades after hemorrhagic or ischemic stroke makes a great contribution to brain damage, mainly are involved in neuronal cell death, blood-brain-barrier (BBB) destruction and development of vasogenic edema. However, the features and direct effect of brain inflammation following stroke is still unknown.

Methods: We adopted the ICH model by injection of collagenase and used a mouse model of transient cerebral ischemia and reperfusion. And pertussis toxin was used to create a pro-inflammatory milieu. Neurodeficits, lesion volume, production of reactive oxygen species (ROS) and inflammatory factors, brain-infiltrating leukocytes and blood-brain-barrier (BBB) destruction were assessed in mice model treated with pertussis toxin or vehicle.

Results: Adopting collagenase induced intracerebral hemorrhage mouse model, we show that pertussis toxin-induced systemic inflammation exacerbated neurological deficits, enlarged lesion size and brain perihematomal edema after intracerebral hemorrhage. Pertussis toxin promoted leukocyte infiltration and inflammatory cytokine release in the brain. Moreover, the integrity of the BBB was further disrupted after receiving pertussis toxin in $\mathrm{ICH}$ mice. Furthermore, we demonstrated that pertussis toxin increased stroke severity and enhanced brain inflammation in middle cerebral artery occlusion (MCAO) mouse model.

Conclusion: Our results suggest that pertussis toxin increases inflammatory response that exacerbates brain injury after intracerebral hemorrhage or ischemic stroke in mouse model.

\section{Background}

Stroke is an acute and severe disease resulting in long-term motor and cognitive neurological deficits, with the characteristics of high morbidity, mortality, disability rate and recurrence rate, including intracerebral hemorrhage (ICH) and ischemic stroke. [1, 2] At present, although progress has been made in understanding the molecular and cellular pathways leading to brain injury after stroke, the current clinical treatments remain poorly effective. ICH accounts for about $10 \%$ to $15 \%$ of all strokes. The damage from $\mathrm{ICH}$ includes not only the primary tissue injury due to the mechanical effects of the hemorrhage but also the development of perihematomal edema (PHE), which induces a severe secondary injury and/or destruction of the adjacent tissue, in addition to an impairment in the integrity of the blood-brain-barrier (BBB).[3-6] Ischemic stroke constitutes $85 \%$ of all strokes. In ischemic brain injury, the neuronal cell death induced by deprivation of glucose and oxygen orchestrates a secondary immune response, which participate in the progression of neuronal damage, BBB disruption and vasogenic edema after ischemia / reperfusion (I/R).[1, 7, 8] Though the primary injury mechanisms differ between acute ischemic stroke and $\mathrm{ICH}$, recent evidence has shown that inflammation contributes to the development and progression of edema, and thus subsequent neurological deterioration in ischemic or hemorrhagic stroke. On the other 
hand, the inflammation after ischemic stroke or $\mathrm{ICH}$ also plays a key role in the tissue reconstruction and brain recovery.[9-12] So whether the direct inflammatory response after stroke is deleterious or beneficial remains a matter of controversies.

Pertussis toxin (PT), an exotoxin produced by Bordetella pertussis, is essential for B. pertussis infection. [13-15] Many lines of evidence show that PT plays an important role in neurological complications of whooping cough. $[16,17]$ And a low-dose PT is usually required to be an immune adjuvant to increase the yield and disease severity in experimental autoimmune encephalomyelitis (EAE), an animal model for multiple sclerosis.[18-20] Mainly because PT enhances permeability of the blood-brain barrier (BBB) and increases migration and infiltration of the inflammatory cells to the central nervous system (CNS).[21, 22] Therefore, the use of PT offers an opportunity to investigate the features and direct effect of systematic inflammation on brain injury following ischemic stroke and hemorrhagic stroke. In this study, we adopted the ICH model by injection of collagenase and used a mouse model of transient cerebral ischemia and reperfusion. We administrated PT to mice and quantified neurological function and brain pathology. Our results provide direct evidence that PT-induced inflammation exacerbated brain injury in hemorrhagic or ischemic stroke.

\section{Materials And Methods}

\section{Animals}

Male C57BL/6 mice were purchased from Charles River (Wilmington, DE, USA). 8- to 10-week-old, 20-25g body weight, age-matched male littermates were used in this study. All mice were randomly assigned to each experiment. All mice were kept in pathogen-free conditions and housed under a 12-h inverted lightdark cycle with access to food and water ad libitum. All surgeries were performed under anesthesia. All animal experiments were approved by the Committee on the Ethics of Animal Experiments of Tianjin Neurological Institute (Tianjin, China) and were performed in accordance with the National Institutes of Health Guide for the Care and Use of Laboratory Animals in China. Reporting of this study complied with the ARRIVE (Animal Research: Reporting in Vivo Experiments) guidelines.[23, 24]

\section{Pertussis toxin administration}

Pertussis toxin (List Biological laboratories, Campbell, CA) was dissolved in distilled-deionized water (DDW) followed by dilution with phosphate buffer saline (PBS) under sterile conditions. And PT was administrated intraperitoneally at a dosage of $250 \mathrm{ng}$ or $500 \mathrm{ng}(1 \mathrm{ng} / \mathrm{ml})$ per mouse immediately after surgery. The same volume PBS was given in the vehicle group.

\section{ICH induction}

$\mathrm{ICH}$ in mouse was induced by intra-striatal injection of collagenase as described previously.[25-27] Mice were anesthetized with an intraperitoneal injection of $5 \%$ chloral hydrate $(30 \mathrm{mg} / \mathrm{kg})$. Briefly, mice were immobilized onto a stereotactic frame and a 1-mm-diameter hole was drilled on the right side of skull (2.3 
$\mathrm{mm}$ lateral to midline and $0.5 \mathrm{~mm}$ anterior to bregma). We injected mice using infusion pump (Hamilton, Reno, NV) into the right striatum with $0.0375 \mathrm{U}$ Type $\otimes$ collagenase (Sigma, St.Louis, MO) in $0.5 \mathrm{ml}$ saline at a depth of $3.7 \mathrm{~mm}$ under the skull. The injection rate was at $0.5 \mathrm{ml} / \mathrm{min}$. Sham mice were subjected to the same surgical procedure with no collagenase injection. Body temperature was maintained at $37.0 \pm$ $0.5^{\circ} \mathrm{C}$ during the surgery.

\section{Middle cerebral artery occlusion (MCAO) procedure}

The model of transient 60 -min intraluminal occlusion of MCA was conducted using the filament method as previously described.[28-30] The mice were anesthetized with $5 \%$ chloral hydrate $(30 \mathrm{mg} / \mathrm{kg}$, intraperitoneal injection). A midline neck incision was then made to expose the left common carotid artery, the external carotid artery and the internal carotid artery, which were all then isolated and ligated. A standardized silicone rubber-coated nylon monofilament (RWD Life Science, Shenzhen, China) was inserted into the the beginning of the left MCA to occlude circulation for $60 \mathrm{~min}$ and the occluding filament was withdraw gently back into the common carotid artery to allow reperfusion. Sham mice were subjected to the same surgical procedure, but the filament was not advanced far enough to occlude the MCA. Cerebral blood flow (CBF) was monitored by a laser Doppler probe (model P10, Moor Instruments, Wilmington, DE, USA) for 5 min both before and after MCAO as well as during the first 10 min of reperfusion. We only included mice that had a residual CBF of $<20 \%$ of the preischemic levels during the ischemic period and recovery levels of $>80 \%$ within $10 \mathrm{~min}$ of reperfusion. The body temperature was maintained at $37.0 \pm 0.5^{\circ} \mathrm{C}$ using an electric warming blanket during the procedures.

\section{Neurological deficit assessment}

Neurological tests were performed by investigators blinded to the treatment groups. The modified Neurological Severity Score (mNSS) test consisted of motor, sensory, reflex and balance assessments with the highest possible score being 18 . The mouse was given one point for the inability to perform each test while deducting one point for the lack of a tested reflex. Finally, an overall score was given to determine impairment in each mouse. The higher score, the more severe impairment from the brain injury. The rota-rod test was performed to evaluate systemic motor function, especially for coordination and balance. The mice were placed on the accelerating rotating rod. The speed was increased from 0 to 40 $\mathrm{rpm}$. Mice were tested 3 times daily with a break of at least $5 \mathrm{~min}$. The lantency to fall off the rotating rod was recorded and the results were calculated as the average of three times.[25, 30, 31]

\section{Neuroimaging}

Magnetic resonance imaging (MRI) was performed using a 7T small-animal MRI (Bruker, Billerica, MA) equipped with a $72 \mathrm{~mm}$ linear transmitter coil and mouse surface receiver coil. The mice were under anesthesia by inhalation of $3.5 \%$ isoflurane, and maintained by $1.0-2.0 \%$ isoflurane in $70 \% \mathrm{~N}_{2} \mathrm{O}$ and $30 \%$ $\mathrm{O}_{2}$. The mice were positioned on a regulated-heated blanket to maintain their body temperatures. The T2weighted images (T2) were performed to assess the lesion volume of $\mathrm{ICH}$ model using the following parameters: repetition time $(T R)=4500 \mathrm{~ms}$, echo time $(\mathrm{TE})=65.5 \mathrm{~ms}$, field of view $(F O V)=28 \times 28 \mathrm{~mm}^{2}$, 
image matrix $=256 \times 256$, slice thickness $=0.5 \mathrm{~mm}$. Susceptibility weighted imaging $(\mathrm{SWI})$ measurements are sensitive to the presence of paramagnetic substances such as iron compounds. The SWI data were obtained with TR $30 \mathrm{~ms}$ and TE $10 \mathrm{~ms}$, flip angle $=25^{\circ}, \mathrm{FOV}=32 \times 32 \times 16 \mathrm{~mm}^{3}$, image matrix $=256 \times 256$. The lesion was drawn using a T2 sequence, and the hematoma was extracted from the SWI sequence with MRIcorN. The hematoma and T2 were transformed to template space by coregistration in SPM8 and overlaid. T2-weighted images of the brain were acquired with a fat-suppressed rapid acquisition with relaxation enhancement sequence $(T R=4000 \mathrm{~ms}, \mathrm{TE}=60 \mathrm{~ms}, \mathrm{FOV}=19.2 \times 19.2$ $\mathrm{mm}^{2}$, matrix size $=192 \times 192$, slice thickness $=0.5 \mathrm{~mm}$ ) to detect the infarct size of the ischemic stroke model. The MRI data were analyzed with Image J software (National Institutes of Health, Bethesda, MD).

To detect reactive oxygen species (ROS) generation in the brain, living bioluminescence images were captured using IVIS spectrum (Perkin Elmer, Waltham, MA). Mice were injected intraperitoneally with 200 $\mathrm{mg} / \mathrm{kg}$ luminol (Invitrogen, Carlabad, CA).[32, 33] Bioluminescence images were captured at $10 \mathrm{~min}$ after injection and the chemiluminescent intensities within the brain were defined and measured. Data were collected as photons per second per $\mathrm{cm}^{2}$ by Living Image Software (Perkin Elmer, Waltham, MA).

\section{Cortical cerebral blood flow (CBF) measurements}

Cortical CBF was monitored by a laser speckle technique, as previously described.[34] Briefly, images were acquired through the laser speckle contrast imager (PeriCam PSI System, Stockholm, Sweden). Mice treated with PT or vehicle were subjected to measurements of CBF at day 3 after MCAO. CBF changes were expressed as a percentage of baseline.

\section{Immunostaining}

The immunostaining was performed as we previously described.[25, 28, 33] Mice were perfused with cold PBS followed by $4 \%$ paraformaldehyde (PFA). Brains were removed, fixed in $4 \%$ PFA and then dehydrated with $15 \%$ sucrose and $30 \%$ sucrose. Next, the isolated brains were embedded in OCT for frozen sections. The frozen slices of $8 \mathrm{~mm}$ thickness were permeabilized and blocked in $5 \%$ donkey serum consisting of $0.3 \%$ Triton $X-100$. Thereafter, tissue sections were incubated with primary antibodies against CD31 (1:50, Abcam, Cambridge, MA), claudin-5 (1:50, Invitrogen, Carlabad, CA) or Matrix Metalloproteinase-9 (MMP-9) $(1: 100, A b c a m, C a m b r i d g e, M A)$ at $4^{\circ} \mathrm{C}$ overnight. After washing with PBS, slices were incubated appropriate fluorochrome conjugated secondary antibodies: donkey anti-rabbit 488 (1:1000, Invitrogen, Carlabad, CA), donkey anti-mouse 546 (1:1000, Invitrogen, Carlabad, CA), at room temperature for $1 \mathrm{~h}$. Nuclei were co-stained with DAPI (Abcam, Cambridge, MA). Images were captured by fluorescence microscope (Model Eclipse 80i, Nikon, Tokyo, Japan). The immunofluorescence intensity was quantified using Image J (National Institutes of Health, Bethesda, MD).

\section{Flow cytometry}

Flow cytometry was performed to analyze immune cell infiltration of brain. [32, 33, 35] Briefly, mice brain tissues were harvested and digested with collagenase囚 (Sigma, St.Louis, MO) to form a single cell 
suspension. After myelin was removed with $30 \%$ percoll (GE Healthcare, Chicago, IL), the cells were stained with fluorochrome conjugated antibodies. All antibodies were purchased from BD Biosciences (San Jose, CA) or BioLegend (San Diego, CA), unless otherwise indicated. The following antibodies were used: CD45 (30-F11), Ly6G (1A8), CD11b (M1/70), CD3 (145-2C11), CD8 (53-6.72), CD4 (GK1.4), NK1.1 (PK136), CD19 (1D3). Flow cytometric measurements were performed on a FACS Aria $(B D$ Biosciences, San Jose, CA) and analyzed using Flowjo 7.6 software (Informer Technologies, Walnut, CA).

\section{Cytokine array}

Inflammatory cytokines in brain tissues were analyzed by Proteome Profiler Mouse XL Cytokine Array. Brain homogenates were prepared from ICH mice treated with or without PT at day 3 after ICH. After the total protein concentration was adjusted to $1 \mathrm{mg} / \mathrm{ml}$, cytokine levels in these samples were detected using a Mouse XL Cytokine Array Kit (R\&D Systems, Minneapolis, MN) according to the manufacturer's instructions.

\section{Statistical analysis}

All results were analyzed by investigators blinded to the treatment groups. And the data are expressed as mean \pm SEM. Statistical data analyses were performed using Graphpad Prism 8.0 software (GraghPad, San Diego, CA). Two-tailed unpaired Student t-test was used to determine the significance of differences between two groups. One-way ANOVA followed by Tukey post hoc test was used for 3 or more groups. Two-way ANOVA accompanied by Bonferroni post hoc test was performed for multiple comparisons. $\mathrm{P}$ values $<0.05$ were considered statistically significant.

\section{Results}

\section{Pertussis toxin augments neurodeficits and brain edema after ICH}

Mice were treated with pertussis toxin (PT, $250 \mathrm{ng}$ or $500 \mathrm{ng}$ ) or vehicle (PBS) by intraperitoneal injection immediately after $\mathrm{ICH}$. To determine the impact of PT on brain injury after $\mathrm{ICH}$, we examined neurological deficits, lesion volume and perihematomal edema in ICH mice receiving PT or vehicle. After ICH induction from day 1 to day 7, PT administration at a dosage of $500 \mathrm{ng}$ aggravated neurodeficits, enlarged lesion size and perihematomal edema as compared to ICH group (Figure 1A-D). These results indicate that 500 ng pertussis toxin administration exacerbates collagenase-induced hemorrhagic brain injury. Thus we adopted $500 \mathrm{ng}$ dose of pertussis toxin for the following investigation.

\section{Pertussis toxin enhances brain inflammation after ICH.}

Reactive oxygen species (ROS) induce oxidative stress, activated alternative death pathways and play an important role in the progression of inflammation. To examine the impact of PT on brain inflammation after $\mathrm{ICH}$, we quantified the ROS production using in vivo bioluminescence at day 1, day 3 and day 7 after $\mathrm{ICH}$. As shown in Figure 2, PT administration induced the production of ROS even in sham mice, suggesting the pro-inflammatory effect of pertussis toxin. ROS levels were significantly higher in ICH 
mice treated with PT relative to vehicle at day 3 and day 7 after ICH (Figure 2A-B). We next assessed the release of inflammatory factors in the brain homogenates of $\mathrm{ICH}$ mice after pertussis toxin administration using a Proteome Profiler Mouse XL Cytokine Array. At $24 \mathrm{~h}$ after ICH surgery, we found that pertussis toxin treatment significantly upregulated the expression of pro-inflammatory cytokines including IL-1, TNF- $a$ and IL-6 (Figure 2C-D). These data show that PT exacerbates ICH induced brain inflammation.

\section{Pertussis toxin promotes brain-infiltrating leukocytes after ICH}

Infiltrating leukocytes are a prominent contributor to brain inflammation and brain damage. To determine the impact of pertussis toxin on leukocyte infiltration, we gated and quantified the numbers of braininfiltrating leukocytes in ICH mice and sham mice treated with PT or vehicle (Figure 3). Pertussis toxin treatment didn't significantly influence immune cell infiltration in sham mice from day 1 to day 7 (Figure 3B). We found infiltrated immune cells after ICH induction, including neutrophils, T cells, B cells and NK cells. Moreover, at day 7 after $\mathrm{ICH}$, brain-infiltrating neutrophils, $\mathrm{CD} 4^{+} \mathrm{T}$ cells and $\mathrm{B}$ cells were further increased in ICH mice treated with $500 \mathrm{ng}$ PT as compared to vehicle (Figure 3B). These data show that PT promotes ICH induced leukocyte infiltration in the brain.

\section{Pertussis toxin aggravates hemorrhagic stroke injury through of BBB breakdown}

Blood-brain-barrier (BBB) dysfunction after ICH play a key role in vasogenic brain edema, perihematomal edema expansion and system immune cell invasion. Meanwhile matrix metalloproteinase-9 (MMP-9) contribute to the further disruption of BBB under inflammatory conditions. $[4,36,37]$ To examine the impact and possible mechanisms of pertussis toxin on the integrity of the BBB after ICH, we quantified tight junction intensity and MMP-9 intensity in brain sections from $\mathrm{ICH}$ mice. As a tight junction protein, claudin-5 loss significantly increased in ICH mice subjected to PT treatment compared with vehicle treated ICH mice (Figure 4A-B). But PT treatment had no significant effect on MMP-9 expression in either group (Figure 4C-D). These data suggest that PT increases the permeability of BBB after ICH mainly through tight junction breakdown. And the BBB destruction caused by PT may contribute to enhanced inflammation and worsen brain injury after $\mathrm{ICH}$.

\section{Pertussis toxin worsens neurological deficits and enlarges infarct lesion after ischemic stroke in mice}

A prominent inflammatory response occurs following not only hemorrhagic but also ischemic stroke, thereby exacerbating secondary injury.[38] To detect the influences of PT on ischemic brain injury, we assessed neurological deficits and ischemic lesion in MCAO mice treated with 500ng PT or vehicle. From day 1 to day 3 after MCAO and reperfusion, the mice treated with PT exhibited higher mNSS score and shorter latency in the rota-rod test compared with controls, as well as enlarged infarct lesion (Figure 5AC). These data suggest that PT also exacerbates acute ischemic brain injury.

Pertussis toxin augments brain inflammation and enhances leukocytes infiltration after brain ischemia. 
To understand the impact of PT on brain inflammation after brain ischemia, we quantified the ROS levels using in vivo bioluminescence at day 3 after MCAO and reperfusion. In sham mice, ROS level was higher in PT group as compared to vehicle. And we found dramatically increased ROS signals in MCAO mice treated with PT relative to vehicle at day 3 after ischemic stroke (Figure 6A-B). These data demonstrate that the pro-inflammatory effect of PT in ischemic brain.

Recruitment of leukocytes occurs in successive waves in the early phase of cerebral ischemia, which significantly influence the pathogenesis of ischemic brain injury.[1] Thus we tested whether PT treatment can impact immune cells infiltration. Flow cytometric results indicated that PT increased the brain infiltration of leukocytes, especially neutrophils, CD $4^{+} T$ cells and B cells in MCAO mice on day 3 after ischemia (Figure $6 \mathrm{C}-\mathrm{D}$ ). These results suggest that PT expands inflammatory milieu of the ischemic brain during the acute stage.

\section{Pertussis toxin aggravates ischemic stroke injury through of BBB destruction}

The initial BBB breakdown occurs $3 \mathrm{~h}$ after ischemic stroke by inflammatory mediators up-regulation and MMPs activation. Therefore claudin- 5 and MMP-9 staining was adopted to identify the effect and the potential mechanism of PT on the permeability of BBB. As shown in Figure 7, MCAO mice treated with PT had increased claudin-5 loss in immunofluorescence intensity within lesion area significantly (Figure 7AB), and which is independent of MMP-9 activation (Figure 7C-D). These data support that the further destruction of BBB caused by PT may promote immune cells migration and exacerbate brain injury after cerebral ischemia.

\section{Discussion}

This study provides evidence that pertussis toxin-induced inflammation exacerbates brain injury after ICH or ischemic stroke. As documented here, pertussis toxin aggravates neurological deficits, lesion size and brain perihematomal edema after $\mathrm{ICH}$. Pertussis toxin increased ROS generation, enhanced BBB permeability, leukocyte infiltration and inflammatory cytokines production in the brain, which may contribute to the aggravated brain injury after $\mathrm{ICH}$. Meanwhile, pertussis toxin plays the similar roles in MCAO model. Pertussis toxin augments brain inflammation through ROS production, BBB destruction and inflammatory accumulation in acute ischemic stroke, that may exacerbate stroke severity. These results suggest that enhanced inflammatory response by pertussis toxin exacerbates brain injury after intracerebral hemorrhage or ischemic stroke.

Mounting evidence have shown that systemic inflammatory response plays a critical role in the secondary injury process of stroke, and the extent of neuronal damage seemed to correlate with the degree of immune response.[9, 39-42] For example, the inhibition of vascular adhesion protein-1 attenuated adhesion and transmigration of circulating immune cells to the site of local injury and ameliorated brain injury during acute phase in ICH and ischemic stroke.[43-45] The sphingosine-1phosphate receptor agonist, siponimod or fingolimod, were confirmed a neuroprotective effect in 
ischemic or hemorrhagic stroke experimentally and clinically, which reduced brain edema and improved neurological outcome by preventing the egress of peripheral lymphocytes from peripheral stores.[46-49] Despite these studies, there is lack of direct evidence about the effect of systemic inflammation on brain injury in the early stage of stroke. In line with previous studies, we show that pertussis toxin-induced systemic inflammation directly exacerbates brain injury in ICH and MCAO mouse model. These results suggest a detrimental impact of systemic inflammatory response at least in the early phase of stroke.

Primary brain injury occurs immediately after the onset of stroke and is often irreversible. In intracerebral hemorrhage, primary brain injury is caused by the tissue disruption due to the parenchymal blood accumulation and the mechanical effect damage associated with the mass effect.[9, 26, 50] In ischemic stroke, the arterial occlusion results in the death of neural cells, engendering an ischemic core, surrounded by a hypoperfused region termed the penumbra. $[1,10]$ Despite the different primary injury mechanisms, the release of damage-associated molecular patterns (DAMPs) defines a common pathway that triggers the innate and adaptive immune response in brain, which induce the secondary brain injury in stroke.[5153] PT mainly contributes to the secondary injury by increasing permeability of BBB and infiltration of the inflammatory cells to CNS. Therefore, PT has the similar effect to brain injury in ICH and ischemic stroke, which was proved by our results.

Inflammation after stroke involves multiple events that include the activation of neuroglia, the infiltration of peripheral leukocytes through disrupted BBB, and the release of inflammatory mediators such as ROS, matrix metalloproteinase (MMPs), cytokines and chemokines. Inflammatory cells and inflammatory mediators have the major role in the pathology of secondary brain damage by exacerbating BBB damage, microvascular failure, brain edema, oxidative stress, and by directly inducing neuronal cell death.[3, 11 , 37] In our study, we found that PT increased the infiltration of systemic immune cells, specifically neutrophils, $\mathrm{CD} 4^{+} \mathrm{T}$ cells and $\mathrm{B}$ cells, to the brain parenchyma. In addition, with the increase of systemic immune cells accumulating in brain, there was a marked increase in proinflammatory cytokines, and an increase in ROS levels. These events in turn led to edema expansion and worsened the clinical outcome during the early phase of stroke.

Inflammatory response not only has influences on the secondary injury of $\mathrm{ICH}$ and ischemic stroke, but also participates in debris clearance, neurotrophin production, and immune regulation. These roles evolve distinctly over time.[4, 11,54] Our results suggest PT-induced inflammatory response exacerbates brain injury during the acute or subacute phase of stroke. However, still unclear are whether and how PT may impact brain recovery after hemorrhagic or ischemic stroke. Few studies address the effect of inflammatory response on long-term outcome during late-stage of stroke. Hirudin, which inhibits the conversion from fibrinogen to fibrin, reduced leukocytes infiltration, modulated microglia phenotype and improved long-term outcome from day 7 to day 28 after $\mathrm{ICH}$, but the benefit of hirudin mainly involves microglia.[55] And lymphocyte infiltration persists during late-stage cerebral ischemia, which probably play some part in the resolution phase.[54,56] Therefore, future studies are required to determine the potential impact of PT-induced inflammatory response in brain recovery of hemorrhagic or ischemic stroke. 


\section{Conclusions}

In summary, our data reveal that PT increases inflammatory response and exacerbates brain injury after $\mathrm{ICH}$ and brain ischemia in mouse model. This study will advance our understanding of brain inflammatory features after hemorrhagic or ischemic stroke, as well as pave the way for immune intervention to benefit stroke patients.

\section{Abbreviations}

C57BL/6: wild type mouse strain used for model induction; ICH: intracerebral hemorrhage; MCAO: middle cerebral artery occlusion; BBB: blood-brain-barrier; PT: pertussis toxin; PHE: perihematomal edema; I/R: ischemia / reperfusion; EAE: experimental autoimmune encephalomyelitis; CNS: central nervous system; DDW: distilled-deionized water; PBS: phosphate buffer saline; CBF: cerebral blood flow; mNSS: the modified Neurological Severity Score; MRI: magnetic resonance imaging; TR: repetition time; TE: echo time; FOV: field of view; ROS: reactive oxygen species; PFA: paraformaldehyde; MMP-9: Matrix Metalloproteinase-9; IL: interleukin; TNF: tumor necrosis factor; DAMPs: damage-associated molecular patterns.

\section{Declarations}

\section{Ethics approval}

All animal experiments were approved by the Committee on the Ethics of Animal Experiments of Tianjin Neurological Institute.

\section{Consent for publication}

Not applicable.

\section{Availability of data and materials}

All data generated or analyzed during this study are included in this published article.

\section{Competing interests}

The authors declared no potential conflicts of interest.

\section{Funding}

This study was supported in part by National Science Foundation of China grant 81771274 .

\section{Author contributions statement}


$\mathrm{MZ}$ and $\mathrm{YL}$ acquired, analyzed and interpreted the data, drafted the manuscript. $\mathrm{HZ}, \mathrm{YF}, \mathrm{YZ}$ and $\mathrm{HL}$ analyzed and interpreted the data. QC, JC, SW and WH analyzed and interpreted the data, edited the language of manuscript. W-NJ formulated the study concept, designed the study, drafted the manuscript and acquired funding for the study.

\section{Acknowledgements}

Not applicable.

\section{References}

1. Jian Z, Liu R, Zhu X, Smerin D, Zhong Y, Gu L, Fang W, Xiong X: The Involvement and Therapy Target of Immune Cells After Ischemic Stroke.Front Immunol 2019, 10:2167.

2. Sternberg Z, Schaller B: Central Noradrenergic Agonists in the Treatment of Ischemic Stroke-an Overview.Trans/ Stroke Res 2020, 11:165-184.

3. Keep RF, Hua Y, Xi G: Intracerebral haemorrhage: mechanisms of injury and therapeutic targets. The Lancet Neurology 2012, 11:720-731.

4. Mracsko E, Veltkamp R: Neuroinflammation after intracerebral hemorrhage. Front Cell Neurosci 2014, 8:388.

5. Shao A, Zhu Z, Li L, Zhang S, Zhang J: Emerging therapeutic targets associated with the immune system in patients with intracerebral haemorrhage (ICH): From mechanisms to translation.EBioMedicine 2019, 45:615-623.

6. Urday S, Kimberly WT, Beslow LA, Vortmeyer AO, Selim MH, Rosand J, Simard JM, Sheth KN: Targeting secondary injury in intracerebral haemorrhage-perihaematomal oedema.Nat Rev Neurol 2015, 11:111-122.

7. Dokalis N, Prinz M: Resolution of neuroinflammation: mechanisms and potential therapeutic option.Semin Immunopathol 2019, 41:699-709.

8. Dong X, Gao J, Zhang CY, Hayworth C, Frank M, Wang Z: Neutrophil Membrane-Derived Nanovesicles Alleviate Inflammation To Protect Mouse Brain Injury from Ischemic Stroke.ACS Nano 2019, 13:12721283.

9. Chen S, Yang Q, Chen G, Zhang JH: An update on inflammation in the acute phase of intracerebral hemorrhage. Transl Stroke Res 2015, 6:4-8.

10. Wang X, Xuan W, Zhu ZY, Li Y, Zhu H, Zhu L, Fu DY, Yang LQ, Li PY, Yu WF: The evolving role of neuroimmune interaction in brain repair after cerebral ischemic stroke.CNS Neurosci Ther 2018, 24:11001114.

11. Zera KA, Buckwalter MS: The Local and Peripheral Immune Responses to Stroke: Implications for Therapeutic Development.Neurotherapeutics 2020, 17:414-435.

12. Zhou Y, Wang Y, Wang J, Anne Stetler R, Yang QW: Inflammation in intracerebral hemorrhage: from mechanisms to clinical translation. Prog Neurobio/ 2014, 115:25-44. 
13. Locht C, Coutte L, Mielcarek N: The ins and outs of pertussis toxin.FEBS J 2011, 278:4668-4682.

14. Melvin JA, Scheller EV, Miller JF, Cotter PA: Bordetella pertussis pathogenesis: current and future challenges. Nat Rev Microbiol 2014, 12:274-288.

15. Nguyen AW, Wagner EK, Laber JR, Goodfield LL, Smallridge WE, Harvill ET, Papin JF, Wolf RF, Padlan EA, Bristol A, et al: A cocktail of humanized anti-pertussis toxin antibodies limits disease in murine and baboon models of whooping cough.Sci Trans/ Med 2015, 7:316ra195.

16. CC. G, EJ. M, A. S, D. B: Pertussis encephalopathy with high cerebrospinal fluid antibody titers to pertussis toxin and filamentous hemagglutinin.Pediatrics 1998, 102:986-990.

17. JH. M, M. K: Workshop on neurologic complications of pertussis and pertussis vaccination.Neuropediatrics 1990, 21:171-176.

18. A. H, T. N, J. Y, M. T, S. K: Analysis of experimental autoimmune encephalomyelitis induced in F344 rats by pertussis toxin administration.neuroimmunology 2000, 104:15-21.

19. JJ. M, IR. M: Adoptive transfer of experimental allergic encephalomyelitis in mice with the aid of pertussigen from Bordetella pertussis. Cellular immunology 1984, 86:541-545.

20. M. A, J. K, Y. L, K. R, Y. K, Y J: Pertussis toxin-induced hyperacute autoimmune encephalomyelitis in Lewis rats is correlated with increased expression of inducible nitric oxide synthase and tumor necrosis factor alpha.Neuroscience letters 2001, 308:41-44.

21. Bruckener KE, el Baya A, Galla HJ, Schmidt MA: Permeabilization in a cerebral endothelial barrier model by pertussis toxin involves the PKC effector pathway and is abolished by elevated levels of cAMP.J Cell Sci 2003, 116:1837-1846.

22. Kugler S, Bocker K, Heusipp G, Greune L, Kim KS, Schmidt MA: Pertussis toxin transiently affects barrier integrity, organelle organization and transmigration of monocytes in a human brain microvascular endothelial cell barrier model.Cell Microbio/ 2007, 9:619-632.

23. Kilkenny C, Browne WJ, Cuthill IC, Emerson M, Altman DG: Improving bioscience research reporting: the ARRIVE guidelines for reporting animal research.PLOS Biol 2010, 8:e1000412.

24. Schulz KF, Altman DG, Moher D, Group C: CONSORT 2010 statement: updated guidelines for reporting parallel group randomised trials.BMJ 2010, 340:c332.

25. Li M, Li Z, Ren H, Jin WN, Wood K, Liu Q, Sheth KN, Shi FD: Colony stimulating factor 1 receptor inhibition eliminates microglia and attenuates brain injury after intracerebral hemorrhage.J Cereb Blood Flow Metab 2017, 37:2383-2395.

26. Zhang J, Shi K, Li Z, Li M, Han Y, Wang L, Zhang Z, Yu C, Zhang F, Song L, et al: Organ- and cellspecific immune responses are associated with the outcomes of intracerebral hemorrhage.FASEB $J$ 2018, 32:220-229.

27. Zhao X, Ting SM, Liu CH, Sun G, Kruzel M, Roy-O'Reilly M, Aronowski J: Neutrophil polarization by IL27 as a therapeutic target for intracerebral hemorrhage.Nat Commun 2017, 8:602.

28. Jin WN, Ducruet AF, Liu Q, Shi SXY, Waters M, Zou M, Sheth KN, Gonzales R, Shi FD: Activation of JAK/STAT3 restores NK-cell function and improves immune defense after brain ischemia. The FASEB 
Journal 2018, 32:2757-2767.

29. Jin WN, Gonzales R, Feng Y, Wood K, Chai Z, Dong JF, La Cava A, Shi FD, Liu Q: Brain Ischemia Induces Diversified Neuroantigen-Specific T-Cell Responses That Exacerbate Brain Injury.Stroke 2018, 49:1471-1478.

30. Yang CS, Guo A, Li Y, Shi K, Shi FD, Li M: DI-3-n-butylphthalide Reduces Neurovascular Inflammation and Ischemic Brain Injury in Mice.Aging Dis 2019, 10:964-976.

31. Zhao H, Feng Y, Wei C, Li Y, Ma H, Wang X, Cui Z, Jin WN, Shi FD: Colivelin Rescues Ischemic Neuron and Axons Involving JAK/STAT3 Signaling Pathway.Neuroscience 2019, 416:198-206.

32. Gan Y, Liu Q, Wu W, Yin JX, Bai XF, Shen R, Wang Y, Chen J, La Cava A, Poursine-Laurent J, et al: Ischemic neurons recruit natural killer cells that accelerate brain infarction.Proc Natl Acad Sci U S A 2014, 111:2704-2709.

33. Jin WN, Shi SX, Li Z, Li M, Wood K, Gonzales RJ, Liu Q: Depletion of microglia exacerbates postischemic inflammation and brain injury.J Cereb Blood Flow Metab 2017, 37:2224-2236.

34. Yang Y, Liu H, Zhang H, Ye Q, Wang J, Yang B, Mao L, Zhu W, Leak RK, Xiao B, et al: ST2/IL-33Dependent Microglial Response Limits Acute Ischemic Brain Injury.J Neurosci 2017, 37:4692-4704.

35. Liu Q, Jin WN, Liu Y, Shi K, Sun H, Zhang F, Zhang C, Gonzales RJ, Sheth KN, La Cava A, Shi FD: Brain Ischemia Suppresses Immunity in the Periphery and Brain via Different Neurogenic Innervations.Immunity 2017, 46:474-487.

36. Min H, Hong J, Cho IH, Jang YH, Lee H, Kim D, Yu SW, Lee S, Lee SJ: TLR2-induced astrocyte MMP9 activation compromises the blood brain barrier and exacerbates intracerebral hemorrhage in animal models.Mol Brain 2015, 8:23.

37. Zhu H, Wang Z, Yu J, Yang X, He F, Liu Z, Che F, Chen X, Ren H, Hong M, Wang J: Role and mechanisms of cytokines in the secondary brain injury after intracerebral hemorrhage.Prog Neurobio/ 2019, 178:101610.

38. Kleinig TJ, Vink R: Suppression of inflammation in ischemic and hemorrhagic stroke: therapeutic options.Current Opinion in Neurology 2009, 22:294-301.

39. Adeoye O, Walsh K, Woo JG, Haverbusch M, Moomaw CJ, Broderick JP, Kissela BM, Kleindorfer D, Flaherty ML, Woo D: Peripheral monocyte count is associated with case fatality after intracerebral hemorrhage.J Stroke Cerebrovasc Dis 2014, 23:e107-111.

40. Dziedzic T: Systemic inflammation as a therapeutic target in acute ischemic stroke.Expert Rev Neurother 2015, 15:523-531.

41. Saand AR, Yu F, Chen J, Chou SH: Systemic inflammation in hemorrhagic strokes - A novel neurological sign and therapeutic target? J Cereb Blood Flow Metab 2019, 39:959-988.

42. Tapia-Perez JH, Karagianis D, Zilke R, Koufuglou V, Bondar I, Schneider T: Assessment of systemic cellular inflammatory response after spontaneous intracerebral hemorrhage.Clin Neurol Neurosurg 2016, 150:72-79. 
43. Ma Q, Manaenko A, Khatibi NH, Chen W, Zhang JH, Tang J: Vascular adhesion protein-1 inhibition provides antiinflammatory protection after an intracerebral hemorrhagic stroke in mice.J Cereb Blood Flow Metab 2011, 31:881-893.

44. Sun P, Hernandez-Guillamon M, Campos-Martorell M, Simats A, Montaner J, Unzeta M, Sole M: Simvastatin blocks soluble SSAO/VAP-1 release in experimental models of cerebral ischemia: Possible benefits for stroke-induced inflammation control.Biochim Biophys Acta Mol Basis Dis 2018, 1864:542-553.

45. Watcharotayangul J, Mao L, Xu H, Vetri F, Baughman VL, Paisansathan C, Pelligrino DA: Postischemic vascular adhesion protein-1 inhibition provides neuroprotection in a rat temporary middle cerebral artery occlusion model.J Neurochem 2012, 123 Suppl 2:116-124.

46. Bobinger T, Manaenko A, Burkardt P, Beuscher V, Sprugel MI, Roeder SS, Bauerle T, Seyler L, Nagel AM, Linker RA, et al: Siponimod (BAF-312) Attenuates Perihemorrhagic Edema And Improves Survival in Experimental Intracerebral Hemorrhage.Stroke 2019, 50:3246-3254.

47. Fu Y, Hao J, Zhang N, Ren L, Sun N, Li YJ, Yan Y, Huang D, Yu C, Shi FD: Fingolimod for the treatment of intracerebral hemorrhage: a 2-arm proof-of-concept study.JAMA Neurol 2014, 71:1092-1101.

48. Sun N, Shen Y, Han W, Shi K, Wood K, Fu Y, Hao J, Liu Q, Sheth KN, Huang D, Shi FD: Selective Sphingosine-1-Phosphate Receptor 1 Modulation Attenuates Experimental Intracerebral Hemorrhage.Stroke 2016, 47:1899-1906.

49. Wei Y, Yemisci M, Kim HH, Yung LM, Shin HK, Hwang SK, Guo S, Qin T, Alsharif N, Brinkmann V, et al: Fingolimod provides long-term protection in rodent models of cerebral ischemia.Ann Neuro/ 2011, 69:119-129.

50. Tschoe C, Bushnell CD, Duncan PW, Alexander-Miller MA, Wolfe SQ: Neuroinflammation after Intracerebral Hemorrhage and Potential Therapeutic Targets.J Stroke 2020, 22:29-46.

51. Fu Y, Liu Q, Anrather J, Shi FD: Immune interventions in stroke.Nat Rev Neuro/ 2015, 11:524-535.

52. Gadani SP, Walsh JT, Lukens JR, Kipnis J: Dealing with Danger in the CNS: The Response of the Immune System to Injury.Neuron 2015, 87:47-62.

53. Taylor RA, Sansing LH: Microglial responses after ischemic stroke and intracerebral hemorrhage.Clin Dev Immunol 2013, 2013:746068.

54. Shi K, Tian D-C, Li Z-G, Ducruet AF, Lawton MT, Shi F-D: Global brain inflammation in stroke. The Lancet Neurology 2019, 18:1058-1066.

55. Li X, Zhu Z, Gao S, Zhang L, Cheng X, Li S, Li M: Inhibition of fibrin formation reduces neuroinflammation and improves long-term outcome after intracerebral hemorrhage./nt Immunopharmacol 2019, 72:473-478.

56. Feng Y, Liao S, Wei C, Jia D, Wood K, Liu Q, Wang X, Shi F-D, Jin W-N: Infiltration and persistence of lymphocytes during late-stage cerebral ischemia in middle cerebral artery occlusion and photothrombotic stroke models. Journal of Neuroinflammation 2017, 14.

\section{Figures}


A

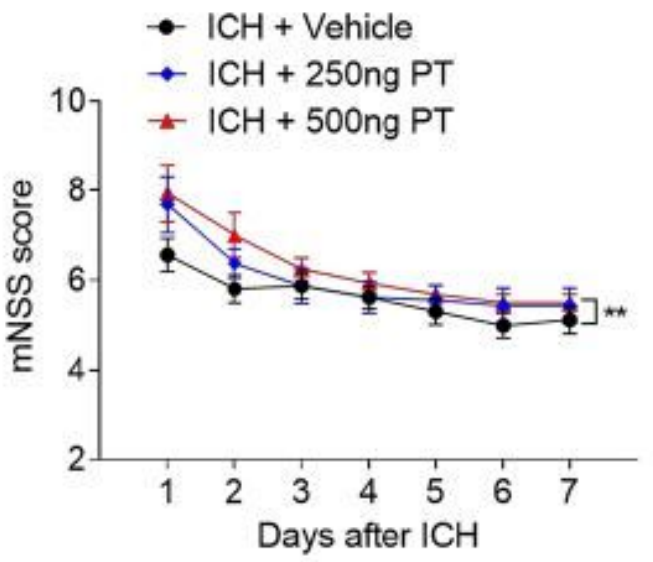

C

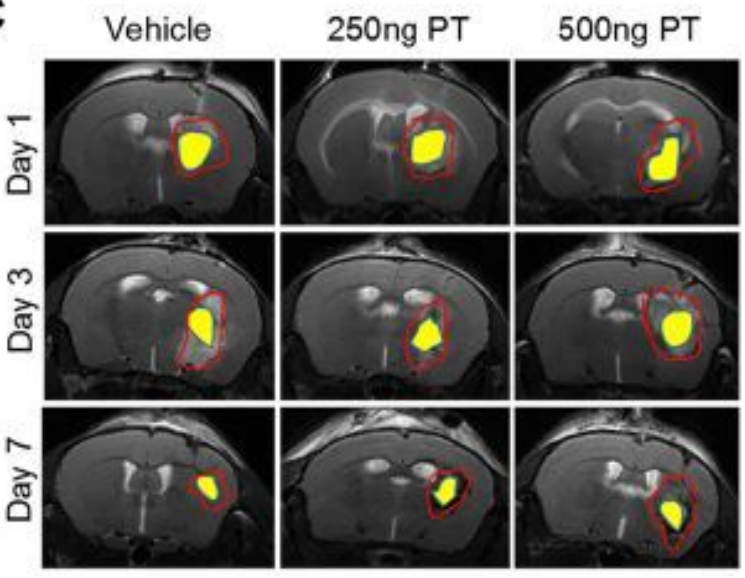

B

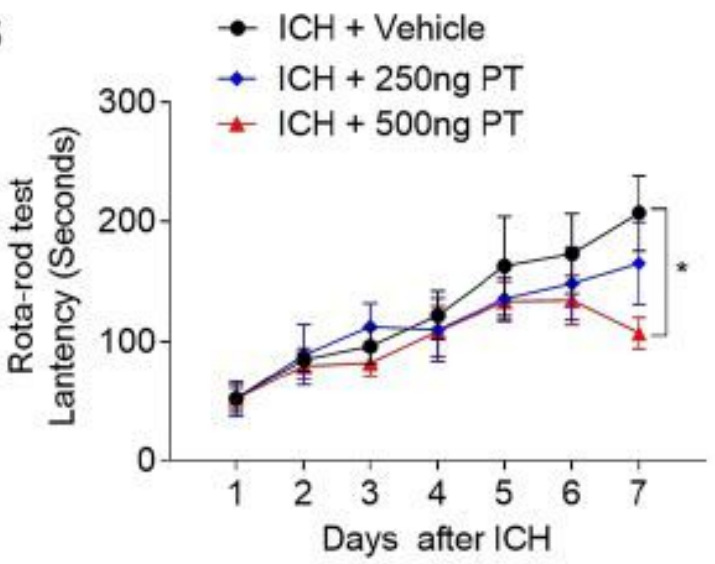

D
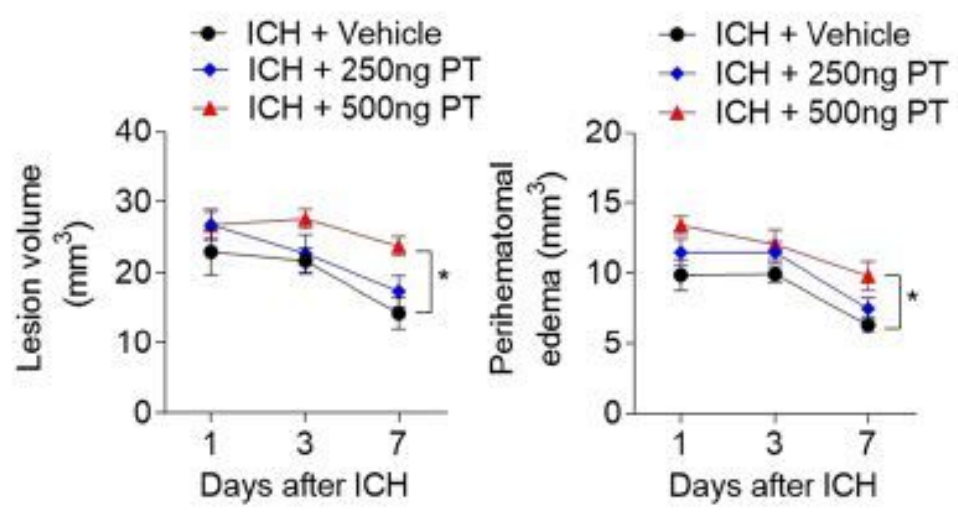

Figure 1

PT increases stroke severity in ICH mice. ICH was induced by injection of $0.0375 \mathrm{U}$ collagenase. After surgeries, mice were treated with PT immediately by intraperitoneal injection at a dosage of $250 \mathrm{ng}$ or $500 \mathrm{ng}$ or vehicle. Mice were subjected to neurological assessment and MRI scanning until day 7 after $\mathrm{ICH}$. A-B. Cumulative data illustrate the neurological assessments of $\mathrm{ICH}$ mice receiving PT or vehicle from day 1 to day 7 after surgery, including mNSS score (A) and rota-rod test (B). $n=8$ mice per group from three independent experiments. Data were expressed as mean $\pm S E M ; * P<0.05 ;{ }^{*} P<0.01$. C. Representative MRI images show lesion area and hematoma area in $\mathrm{ICH}$ mice receiving PT or vehicle. Red lines delineate lesion area, yellow shadows represent hematoma area, and perihematomal edema volume was calculated by subtracting the hematoma volume from lesion volume. D. Cumulative data show the lesion volume and perihematomal edema volume of $\mathrm{ICH}$ mice receiving PT or vehicle. $\mathrm{n}=4$ mice per group from two independent experiments. Data were expressed as mean $\pm S E M$; ${ }^{*} P<0.05$. 
A

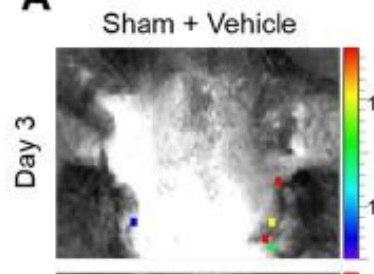

Sham + 500ng PT
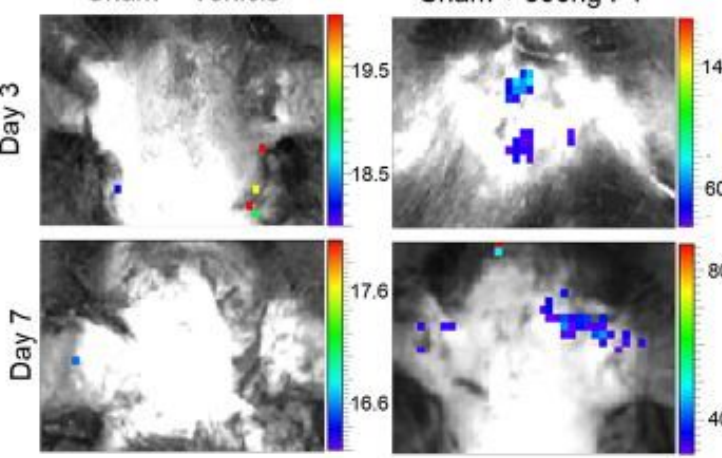

C

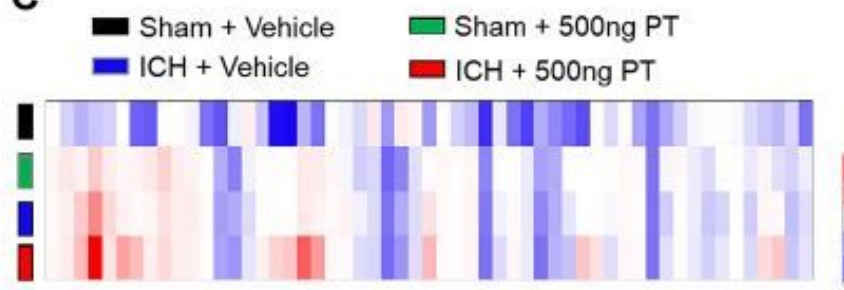

$\mathrm{ICH}+$ Vehicle

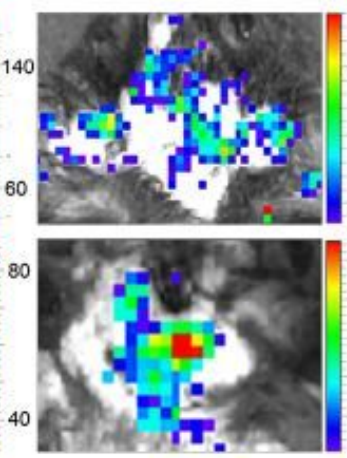

D

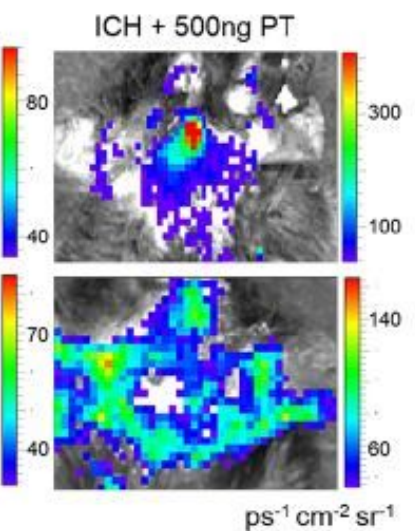

$\mathrm{ps}^{-1} \mathrm{~cm}^{-2} \mathrm{sr}^{-1}$
B

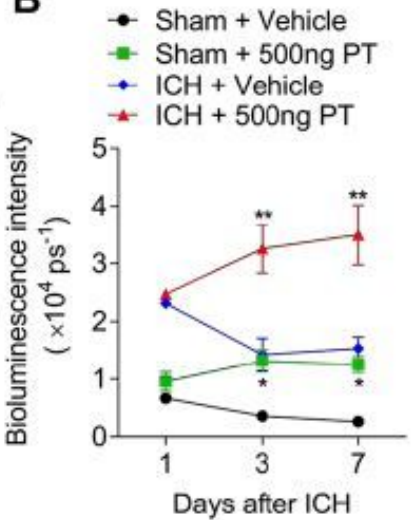

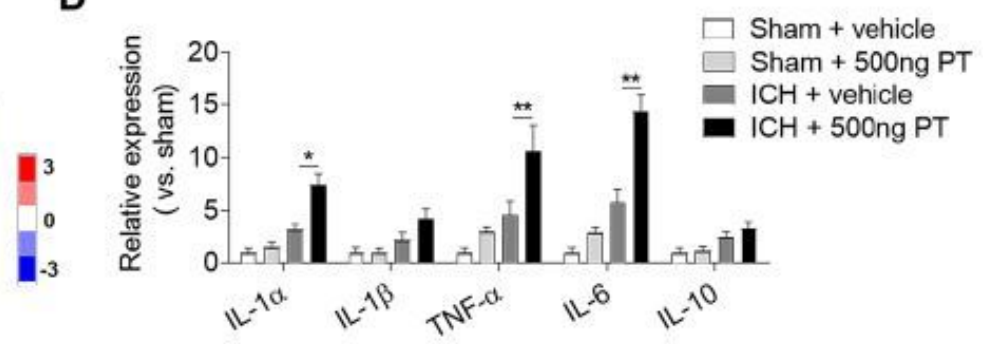

Figure 2

Pertussis toxin enhances brain inflammation after ICH. ICH in mice was induced using $0.0375 \mathrm{U}$ collagenase injection. PT treatment was given immediately after ICH by intraperitoneal injection, at a dosage of $500 \mathrm{ng}$. A-B. At day 1 , day 3 and day 7 after $\mathrm{ICH}$, representative bioluminescence images and quantification analysis show ROS generation in sham and ICH mice receiving PT or vehicle. $\mathrm{n}=3$ mice per group from two independent experiments. C. Brain tissues were obtained from ICH mice receiving pertussis toxin or vehicle. Sham operated mice receiving pertussis toxin or vehicle were used as control. Brain homogenates were analyzed by a Mouse XL Cytokines Array kit. Heat map and cluster analysis show the expression of inflammatory factors in brain homogenates from sham and $\mathrm{ICH}$ mice with indicated treatments. A heat map was generated and the relative pixel intensity of spots signal is indicated by the representative color code (red, upregulated; blue, down-regulated). D. Bar graphs show the top significantly dysregulated factors. $n=6$ mice per group. The data were calculated as mean \pm SEM; ${ }^{*} \mathrm{P}<0.05 ;{ }^{* *} \mathrm{P}<0.01$. 
A

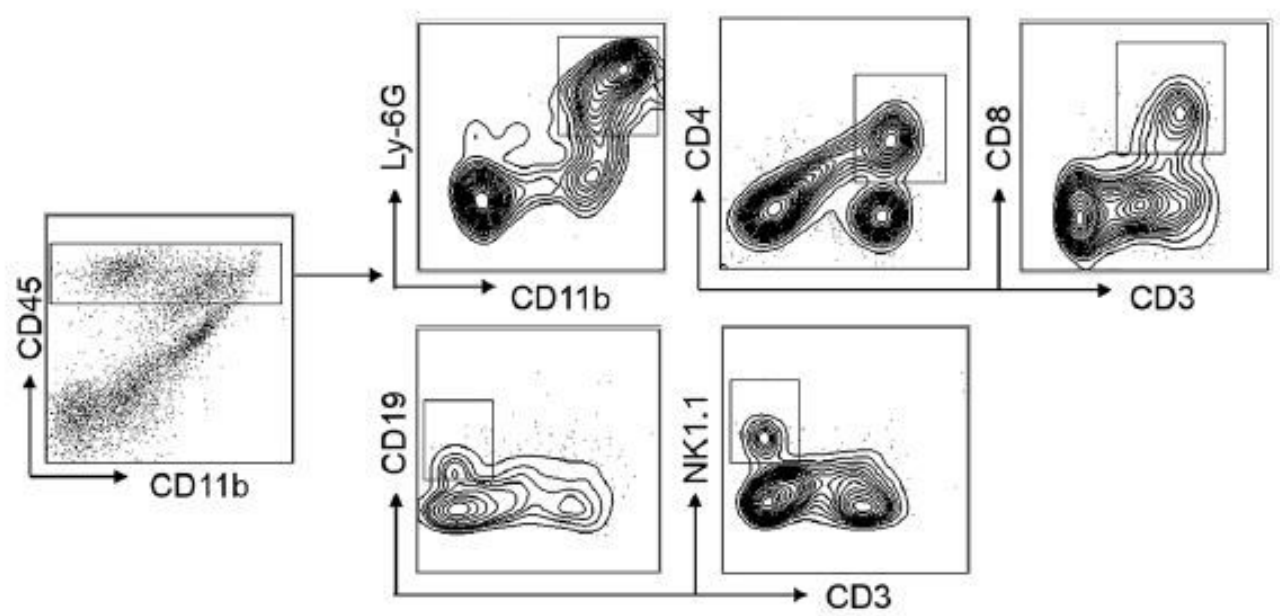

B $\rightarrow$ Sham + Vehicle - Sham + 500ng PT $\rightarrow \mathrm{ICH}+$ Vehicle $+\mathrm{ICH}+500$ ng PT

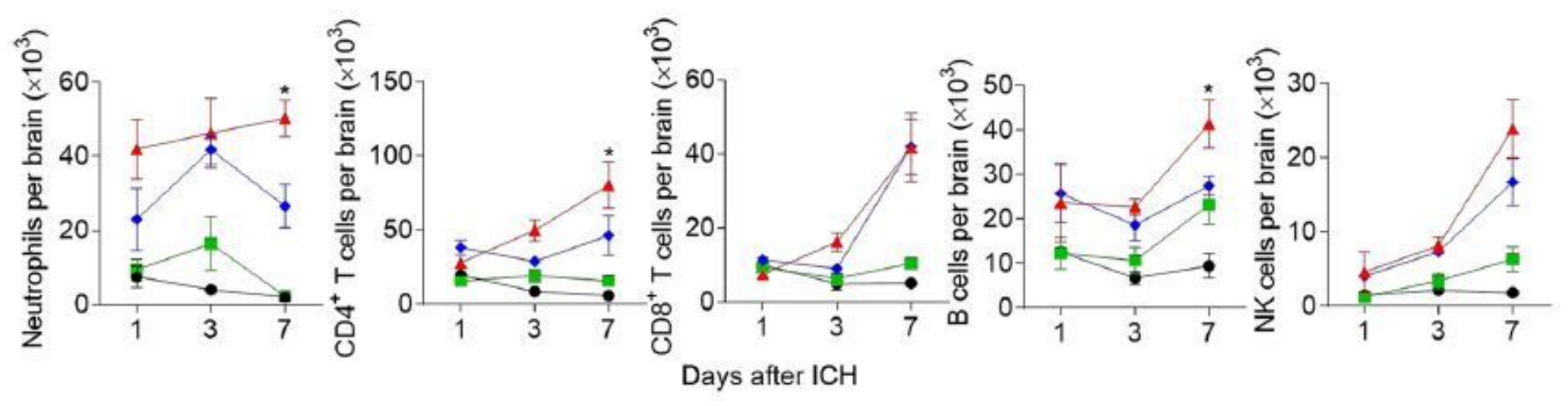

Figure 3

PT promotes brain-infiltrating leukocytes after $\mathrm{ICH}$. ICH was induced by $0.0375 \mathrm{U}$ collagenase injection. At day 1 , day 3 or day 7 after $\mathrm{ICH}$, single cell suspensions were isolated from brain tissues of ICH mice treated with PT or vehicle. A. Gating strategy of brain-infiltrating immune cells including neutrophils (CD45highCD11b+Ly6G+), CD4+ T cells (CD45highCD3+CD4+), CD8+ T cells (CD45highCD3+CD8+), B cells (CD45highCD3-CD19+), and NK cells (CD45highCD3- NK1.1+). B. Quantification of lymphocytes, monocytes and neutrophils in the brain of sham and ICH mice receiving PT or vehicle at indicated time points. Day 1 after $\mathrm{ICH}, \mathrm{n}=3$ mice per group from two independent experiments; Day 3 and Day 7 after $\mathrm{ICH}, \mathrm{n}=5$ mice per group from three independent experiments. The data were calculated as mean $\pm \mathrm{SEM}$; $* \mathrm{P}<0.05$. 


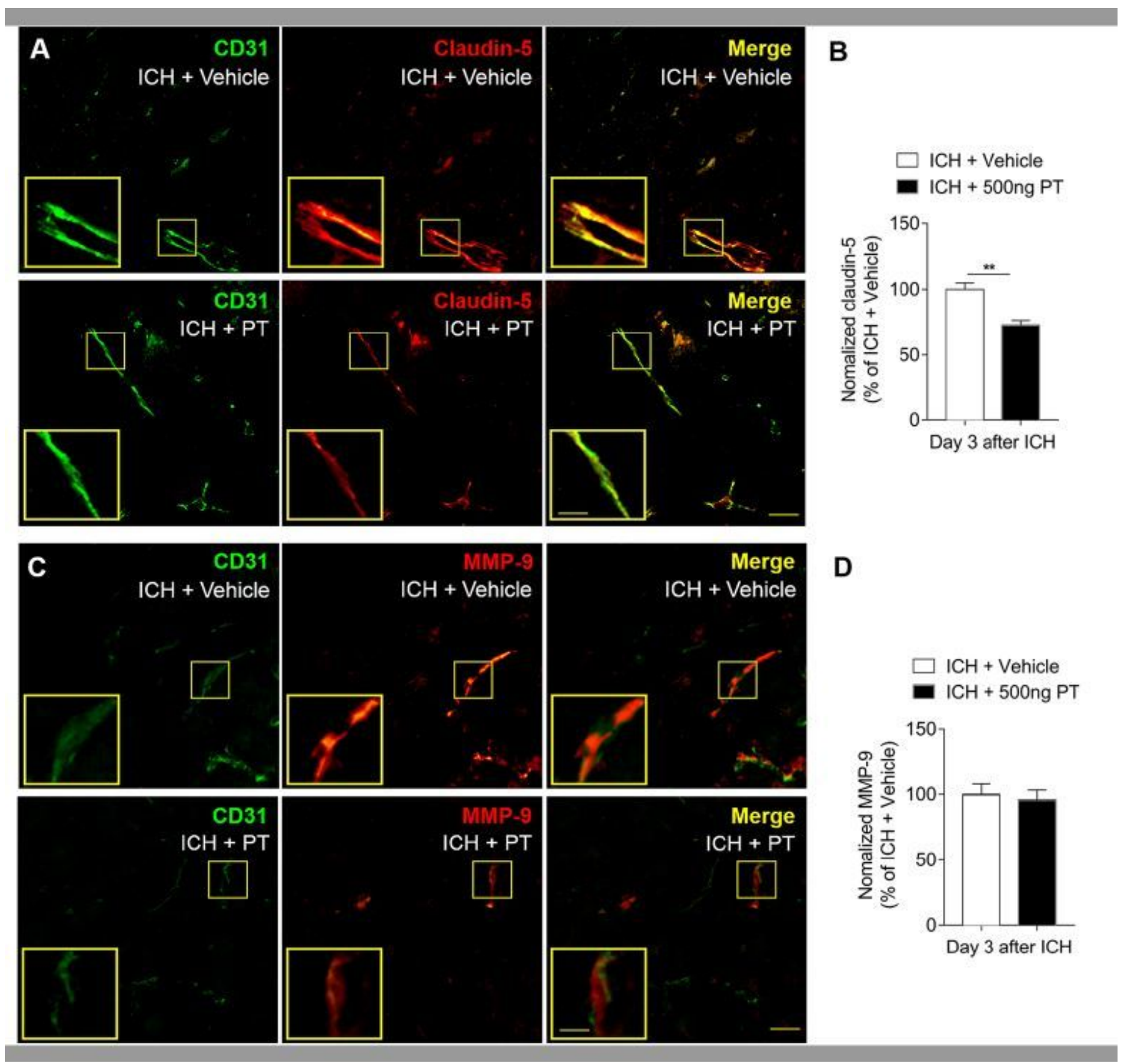

\section{Figure 4}

PT increases the permeability of blood-brain barrier after $\mathrm{ICH}$. ICH was induced by $0.0375 \mathrm{U}$ collagenase injection. A. Fluorescent images show immunostaining of CD31 (green) and claudin-5 (red) in brain sections from ICH mice treated with 500 ng PT or vehicle at day 3 after ICH. Scale bar: $25 \mu \mathrm{m}$, insert: $10 \mu \mathrm{m}$. B. Bar graphs show that ICH mice treated with $500 \mathrm{ng}$ PT had increased claudin-5 loss in immunofluorescence intensity in lesion area. C. Brain sections from ICH mice treated with PT or vehicle were stained with CD31 (green) and MMP-9 (red) at day 3 after ICH. Scale bar: $25 \mu \mathrm{m}$, insert: 10 $10 \mathrm{~m}$. D. Quantitative analysis shows that PT had no significant effect on the expression of MMP-9 in 
immunofluorescence intensity in lesion area. $\mathrm{n}=3$ mice per group from three independent experiments. Data were expressed as mean \pm SEM; ${ }^{* *} \mathrm{P}<0.01$.

A
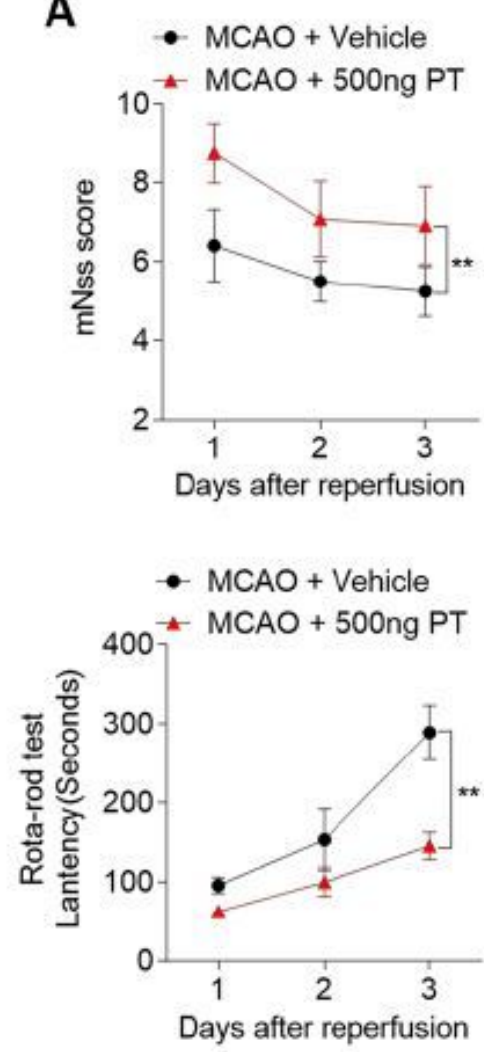

B

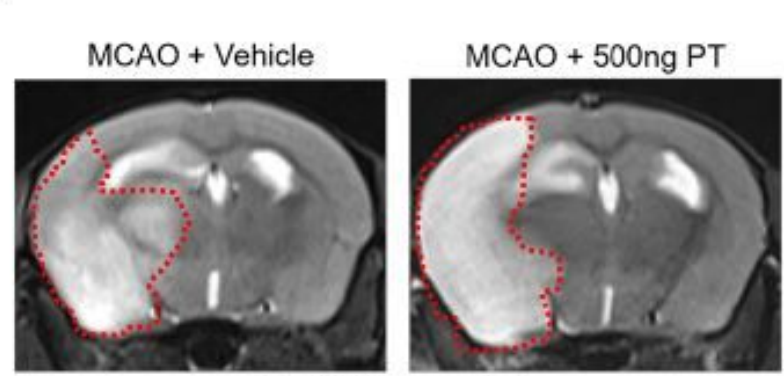

Day 3 after reperfusion

C

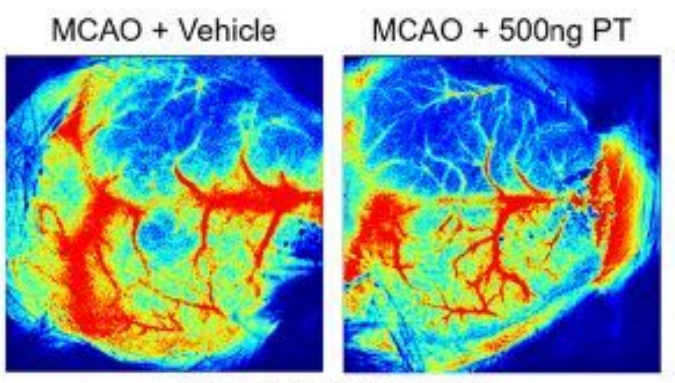

Day 3 after reperfusion
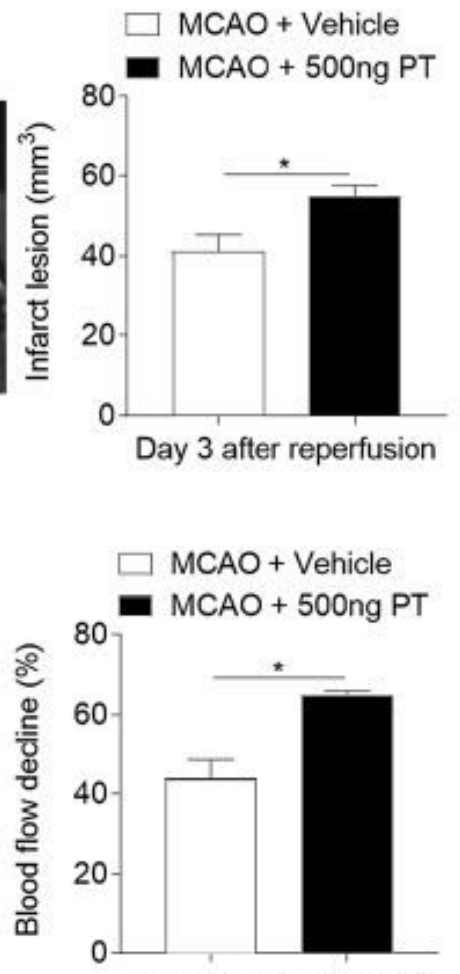

Day 3 after reperfusion

\section{Figure 5}

PT exacerbates neurological deficits and brain infarction after cerebral ischemia and reperfusion. Mice were treated with 500ng PT or vehicle immediately by intraperitoneal injection after 60 min MCAO and reperfusion. Neurological deficits, lesion volume and the cerebral blood flow (CBF) were evaluated in MCAO mice. A. Summarized results show the mNSS scores and the rota-rod test lantency of MCAO mice receiving $\mathrm{PT}$ or vehicle at the indicated times after reperfusion. $n=6$ mice per group from three independent experiments. B. Representative MRI images show infarct area (outlined in red) in MCAO mice receiving $\mathrm{PT}$ or vehicle after reperfusion. Bar graphs show the infarct lesion at day 3 after ischemia/reperfusion. $\mathrm{n}=4$ mice per group. $\mathrm{C}$. Images of CBF in MCAO mice that received PT or vehicle treatment. Quantification of reduced blood flow in the ipsilateral hemisphere. The data were calculated as mean \pm SEM; ${ }^{*} \mathrm{P}<0.05,{ }^{*} \mathrm{P}<0.01$. 


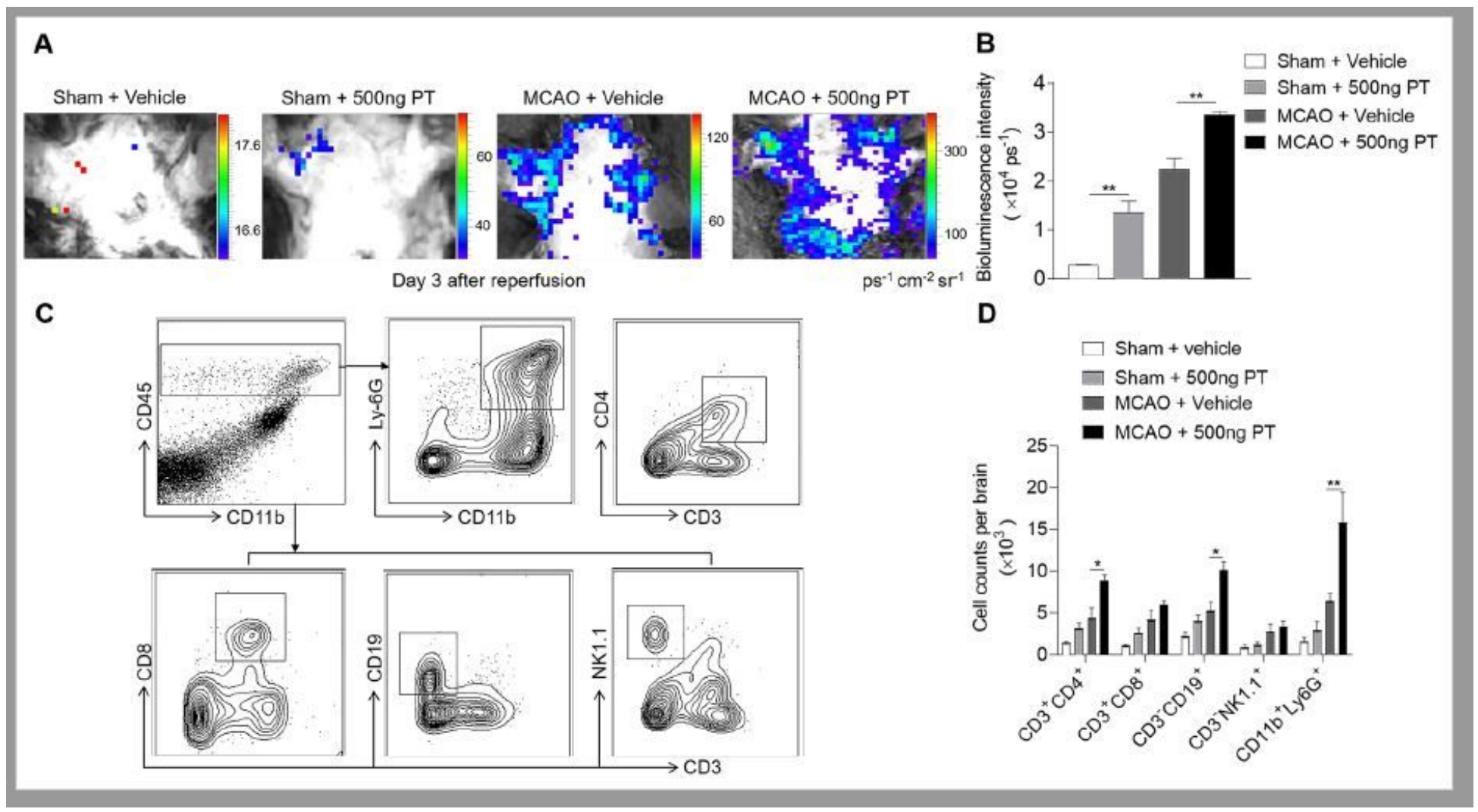

Figure 6

PT augments brain inflammation after brain ischemia and reperfusion. A-B. Visualization of ROS generation in vivo bioluminescence imaging and quantification of signal strength in MCAO mice given PT or vehicle at day 3 after ischemia and reperfusion. $n=3$ mice per group from two independent experiments. C. Counts of CNS-infiltrating immune cell subsets were measured using flow cytometry on day 3 after reperfusion. Representative flow cytometry plots show the gating strategy of leukocyte subpopulations isolated from the brain. D. Summarized results show the cells counts of the indicated subsets in the brain of sham and MCAO mice receiving PT or vehicle. $\mathrm{n}=4$ mice per group. The data were calculated as mean $\pm \mathrm{SEM} ;{ }^{*} \mathrm{P}<0.05,{ }^{*} \mathrm{P}<0.01$. 


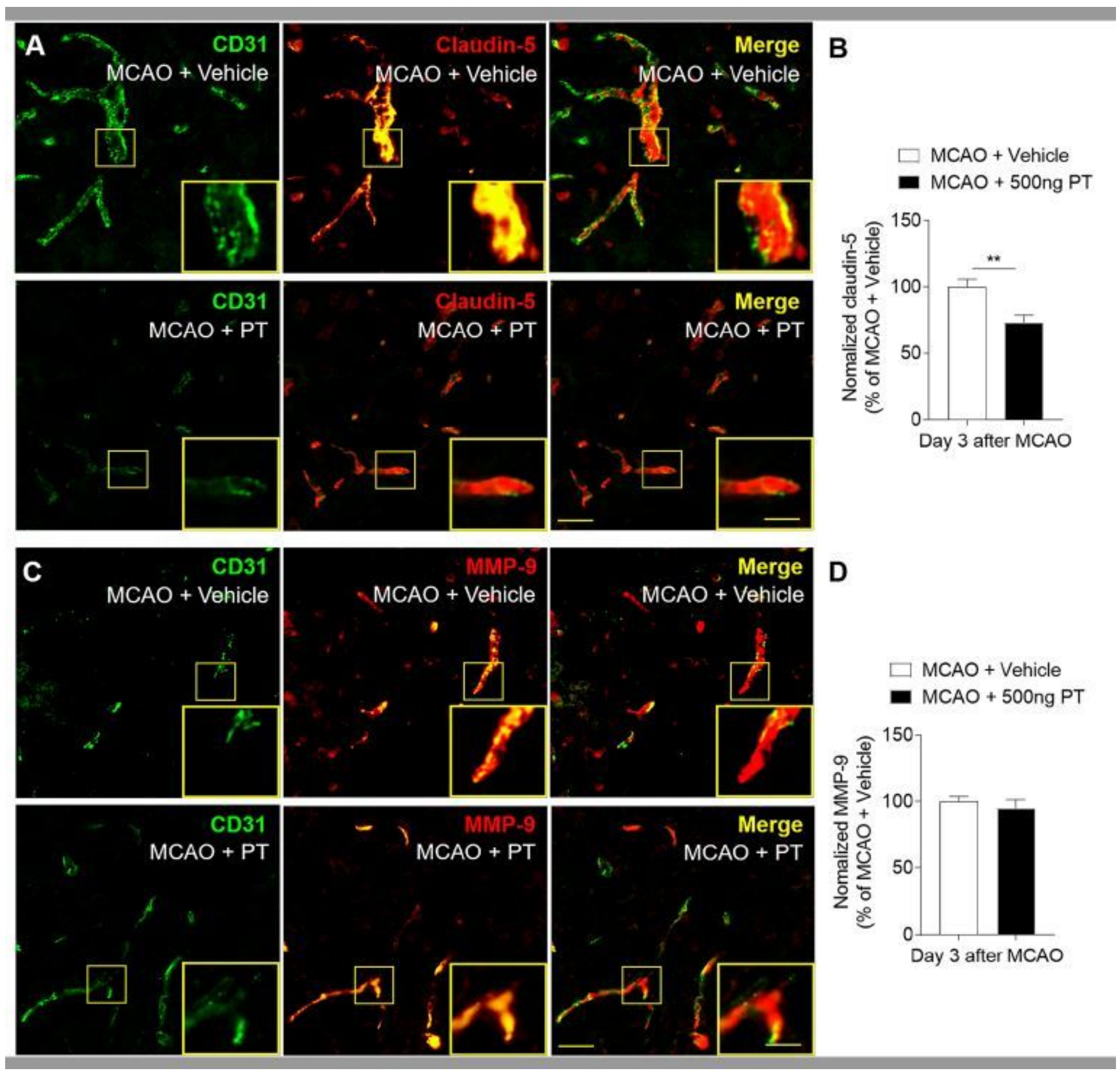

Figure 7

PT enhances the permeability of blood-brain barrier after ischemia. A. Representative images show staining of CD31 (green) and claudin-5 (red) in brain sections from MCAO mice at day 3 after ischemia and reperfusion. Scale bar: $25 \mu \mathrm{m}$, insert: $10 \mu \mathrm{m}$. B. Quantification of claudin-5 loss in immunofluorescence intensity after exposure to indicated treatment. $n=5$ mice per group from three independent experiments. C. Immunostaining of CD31 (green) and MMP-9 (red) in brain sections from MCAO mice receiving PT or vehicle. Scale bar: $25 \mu \mathrm{m}$, insert: $10 \mu \mathrm{m}$. D. Summarized results show that PT 
had no influence on the production of MMP-9 in intensity in lesion area. $n=16$ sections from 5 mice in each group. The data were calculated as mean $\pm \mathrm{SEM} ; * \star \mathrm{P}<0.01$. 